\title{
Room Temperature Chemical Vapor Deposition for Fabrication of Titania Inverse Opals: Fabrication, Morphology Analysis and Optical Characterization
}

\author{
Jun Hyuk Moon, 'Young-Sang Cho, and Seung-Man Yang* \\ Deparment of Chemical and Biomolecular Engineering, Sogang Universitv. Seoul 121-742, Korea \\ ${ }^{*}$ E-mail: junhwhiasogang ack \\ Department of Chemical and Biomolecular Engineering. Korea Atwanced Institute of Science and Technologv, \\ Daejeon 305-701, Korea \\ Received Juty 6, 2009, Accepted August 9, 2009
}

\begin{abstract}
This paper demonstrates room temperature chemical vapor deposition (RTCVD) for fabricating titania inverse opals. The colloidal cry stals of monodisperse poly'mer latex spheres were used as a sacrificial template. Titania was deposited into the interstices between the colloidal spheres by alternate exposures to water and titanium tetrachloride $\left(\mathrm{TiCl}_{4}\right)$ vapors. The deposition was achieved under atmospheric pressure and at room temperature. Titania inverse opals were obtained by buning out the colloidal template at high temperatures. The filling fraction of titania was controlled by the number of deposition of $\mathrm{TiCl}_{4}$ vapor. The mophology of inverse opals of titania were investigated. The optical reflection spectra revealed a photonic band gap and was used to estimate the refractive index of titania.
\end{abstract}

Key Words: Chemical vapor depostision. Titania inverse opals. Photonic band gap

\section{Introduction}

Three-dimensional (3D) ordered porous structures have been used in a wide range of applications from optoelectronics to photoelectrochemistry. Specifically. 3D titania structures have attracted particular interest due to their unique optical and physicochemical properties. These properties are attractive for many applications, including visible photonic crystals, ${ }^{1.2}$ chemical and biological sensors. ${ }^{3}$ tunable superhy drophobic surfaces $^{+}$and photochemical electrodes in solar cells. ${ }^{-\infty}$

In general, the formation of 3D titania structures has been achieved by sacrificial templating method. In this method. preformed structures are used as a template for precursor deposition, which is followed by the removal of the template to reveal a $3 \mathrm{D}$ titania structure. The various approaches used to fabricate 3D templates include colloidal assembly. directwriting. layer-by'-layer photolithography. ${ }^{10}$ and multi-beam interference lithography. ${ }^{1}$ Among these, the self-assembly of colloidal particles is a novel and cost-effective approach. Additionally, colloidal self-assembly is attractive in that the lattice constants and pore size of the colloidal cristals can be easily controlled by changing the particle size. ${ }^{1.213}$ Meanwhile. deposition into 3D templates has been achieved by various methods. including infiltration of a precursor solution ${ }^{1.1+1.1 \%}$ or dispersion of nanoparticles. ${ }^{12.18}$ liquid-phase deposition in a supersaturated precursor solution. ${ }^{19}$ and chemical vapor deposition.

In this paper. we present a simple titania vapor-deposition method. performed under atmospheric pressure and at room temperature. to fabricate inverse titania opals. Vapor-phase deposition has several advantages over deposition by liquidphase precursors. Specifically, it gives a more uniform and controlled infiltration over a large area in relatively short times. Recently. King and coworkers demonstrated atomic layer deposition (ALD) of titania using a vapor-phase titania pre- cursor. $^{2(1)}$ They performed a precise deposition (less than $1 \mathrm{~nm}$ thickness resolution) onto colloidal silica crystals at around $100^{\circ} \mathrm{C}$. Compared to this result, the deposition conditions used in the present study allow the use of poly meric opal templates. Therefore control of the morphology of the inverse structures can be further achieved by annealing ${ }^{21}$ or etcling of the polymeric opal templates. $\stackrel{2}{ }$ and allows fine tune of photonic bandgap properties.

In this study. the morphology of the resulting inverse titania opals was characterized by scanning electron microscopy (SEM). The filling fraction and the crystalline phase of titania were measured by thermal gravitational analy'sis (TGA) and $\mathrm{X}$-ray diffraction $(\mathrm{XRD})$, respectively. The optical reflection was used to locate a photonic bandgap of the inverse titania opals and estimate the refractive index of the titania.

\section{Experimental Section}

Materials. Monodisperse polystyrene (PS) latex particles were synthesized by enulsifier-free emulsion polymerization using sty rene (Showa. $98 \%$ ) as the monomer. potassium persulfate (Aldrich) as the initiator. potassium bicarbonate (Sigma) as the buffer and sodium styrene sulfonate (Aldrich) as the comonomer. The diameter of the PS spheres was $260 \mathrm{~nm} \pm 3$ $\mathrm{nm}$, measured from SEM images. Micrometer-sized PS spheres (I $\mu \mathrm{m}$ in diameter) were purchased from Interfacial Dy namics. These particles have sulfonate groups on the surface for electrostatic stabilization in aqueous colloids. In the later stage of colloidal templating. the sulfonate groups produce a strong adhesion between the latex surface and the infiltrated titania as a result of electrostatic attraction. ${ }^{23}$

Fabrication of colloidal crystal film. The PS colloidal spheres of $260 \mathrm{~nm}$ diameter were dip-coated on a fused glass substrate or silicon wafer. To obtain a clean hydrophilic substrate. the substrate was washed with an organic solvent and treated with 
oxygen plasma. In the dip-coating, the substrate was placed vertically into about $5 \mathrm{wt} \%$ colloidal suspension and lifted at a speed of $0.5 \mu \mathrm{m} / \mathrm{s}$. For the colloidal crystals of the micrometersized PS particles, we used an alternative to dip-coating due to the fast sedimentation of particles. The colloidal crystal films were assembled by using capillary-induced injection of the PSS colloid into a narrow region between two glass substrates, which were spaced with a pre-patterned photoresist. The thickness of the prepared colloidal crystal films was in the range of around $15 \mu \mathrm{m}$.

Fabrication of titania inverse opals. The deposition of titania was achieved in a batch reactor under atmospheric pressure and at room temperature. Titanium tetrachloride ( $\mathrm{TiCl}_{4}$, Aldrich) was used as the titania precursor ${ }^{24.25}$ (Caution: titanium tetrachloride is a very corrosive liquid. When in contact with the moisture in the air, it reacts to give off hydrogen chloride fumes, which are very toxic. Use it only with adequate ventilation and wear protective clothing and safety goggles.) The colloidal crystal film was exposed alternately to water vapor for $30 \mathrm{~min}$ and subsequently to $\mathrm{TiCl}_{4}$ vapor for $10 \mathrm{~min}$. The concentration of $\mathrm{TiCl}_{+}$was about $0.03 \mathrm{vol} \%$ in moisture-free argon gas and water vapor in $60 \%$ relative humidity. The sacrificial PS colloidal crystal templates were removed by calcinations at $500^{\circ} \mathrm{C}$ at 2 " $\mathrm{C} / \mathrm{min}$ for $3 \mathrm{~h}$ in air.

Characteeization. Thermal gravitational analysis (TGA, TA instrument TGA-Q500) was used to confirm the formation of titania inverse opals during the calcination and estimate the filling ratio of titania. For the images of the prepared colloidal crystals and their inverted structures, we used a scanning electron microscope (SF:M, Philips XI.30). Before the SFM imaging, all samples were coated with Au to make them conductive. The SLM images were also used to estimate the size distributions of the PS particles and their inverted pores by averaging $100-200$ particles and pores, respectively. The optical reflection was measured with a UV-Vis spectrophotometer. The crystalline phase was examined with a powder $\mathrm{X}$-ray diffractometer with $\mathrm{Cu} \mathrm{Ka}$ radiation (XRD, Rigaku D/MAX-RC)

\section{Results and Discussion}

The PS particles were self-assembled into a facc-centered cubic lattice with the (1II) planes facing toward the substrate (Figure la). The dried colloidal crystal film was exposed to water vapor and precursor vapor in sequence. The $\mathrm{TiCl}_{4}$ vapor then reacted on the moistened particle surface of the colloidal crystal, producing titania ria the sol-gel synthetic route. The $\mathrm{TiCl}_{4}$ was then hydrolyzed by nucleophilic attack of water molecules on the particle surface, and the hydrolyzed precursor was transformed into titania via polycondensation. After titania deposition, the composite colloidal crystal film was calcined at $500^{\circ} \mathrm{C}$ to obtain inverse titania opals. Figures $\mathrm{Ib}$ shows representative a SEM image of the inverse titania opals produced from the colloidal crystals of $260 \mathrm{~nm}$ PS spheres. The linear volume shrinkage was measured to be about $20 \%$, which is similar to previous results. ${ }^{15.17 .26 .27}$

Figure 2 compares SEM images of inverse titania opals templated from $260 \mathrm{~nm}$ and $1 \mu \mathrm{m}$ PS spheres. The magnified

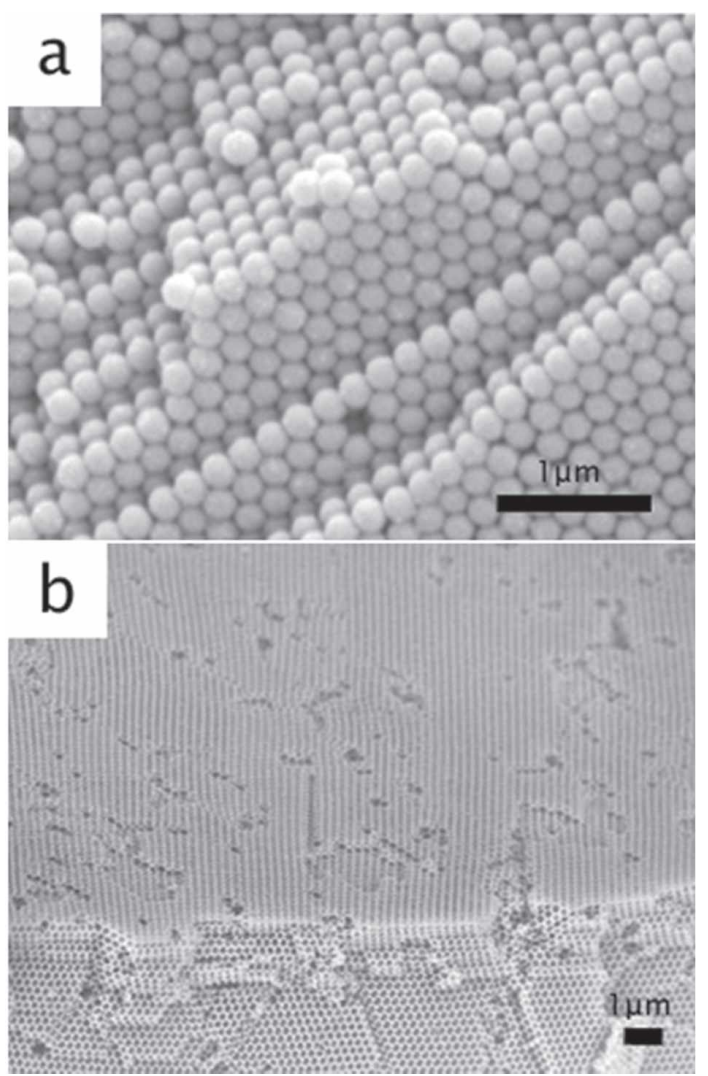

Figure 1. SEM images of (a) PS colloidal crystals and (b) titana inverse opals.

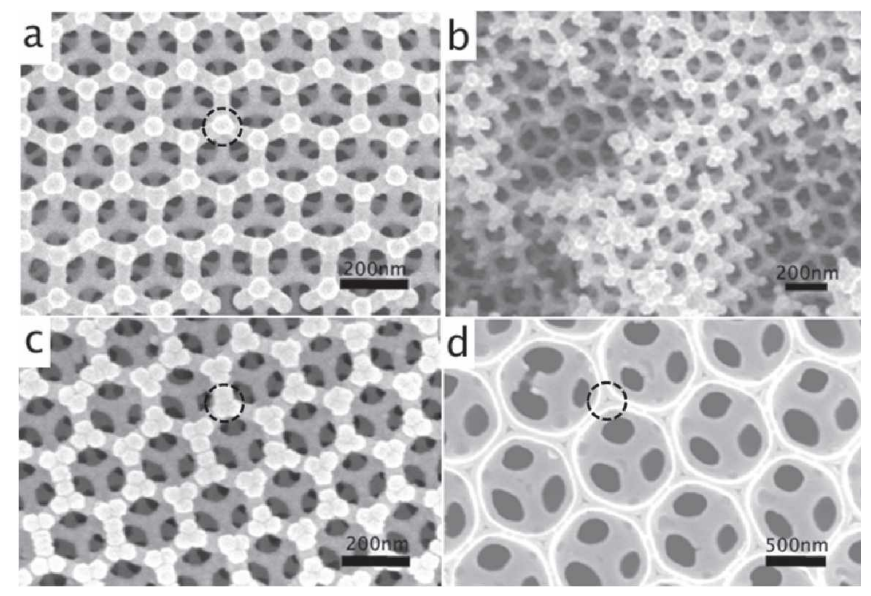

Figure 2. SEM images of tiania inyerse opals prepared from the collordal crs stal template of 260 ) $\mathrm{mm}$ PS spheres with (a.b) single and (c)double deposition of TiCl. sapor. (d) SEM Images of utana Inverse opal produced from the colloidal crystal template of I $\mu \mathrm{m}$ PS spheres with single deposition. The dotted circles midicate (a.c) rod-like shell and (d) conformally coated shell.

images of top-view and internal structures reveal that the titania layer coated on the $260 \mathrm{~nm}$ particles shrank into a rod-like morphology (Figures 2a and 2b), while the coated layer formed a shell in the case of $1 \mu \mathrm{m}$ particles (Figure $2 \mathrm{~d}$ ). It has been found previously that inverted opals can exist as several different structures, including completely filled opals, 
opal shells with air core, and skeletal opal structures with rod-like shells. depending on the mechanical stability of the coated materials and the interfacial energy between the template and the coating materials. ${ }^{78.29}$ Moreover, the morphology of the shell has the effect on photonic bandgap properties. It has been shown that inverse opals with a dielectric shell possess larger photonic bandgap width than completely filled structures, and skeletal inverse opals have multiple photonic bandgaps. ${ }^{14,31}$

In the case of the present stidy. the difference in morphology can be attributed to a partially condensed titania matrix and the surface tension on the matrix by the interstitial space. Since the deposition occurs at room temperature. the resulting titania contains an aqueous moiety and uncondensed hydroxỵl groups. leaving gel-like shells. This was confinmed by TGA in which a $\sim 10 \%$ weight loss was observed at $100^{\circ} \mathrm{C}$. due to the esorption of the aqueous moleties and dehydration of the titania matrix. Therefore. the shell might be soft enough to be defonnable. Meanwhile for colloidal cry stals of smaller spheres. a ligher surface tension can be exerted on the gel-like coated layer due to ligher curvature. Therefore, duning heat treatment of the colloidal crystal of the $260 \mathrm{~nm}$ PS spheres. reformation of the shells into a rod-like morphology is likely to occur.

We controlled the film thickness by the number of $\mathrm{TiCl}_{4}$ and water vapor depositions. A single deposition consists of two exposures of both water vapor and precursor vapor. With the colloidal cry stals of $260 \mathrm{~nm}$ PS spheres. the coated titania layer had a thickness (diameter of cylindncal wall) of about $60 \mathrm{~nm}$ after the first deposition (Figures 2a. 2b). One additional deposition increased the thickness to $80 \mathrm{~nm}$ (Figure 2c): however after this deposition. the samples were almost completely covered with the film and no subsequent increases in the film thickness were measured. The average growth rate was about $30 \mathrm{~nm}$ per deposition. More precise deposition might be acheved by decreasing the exposure time and the concentration of the precursor vapor. Meanwhile. inverse

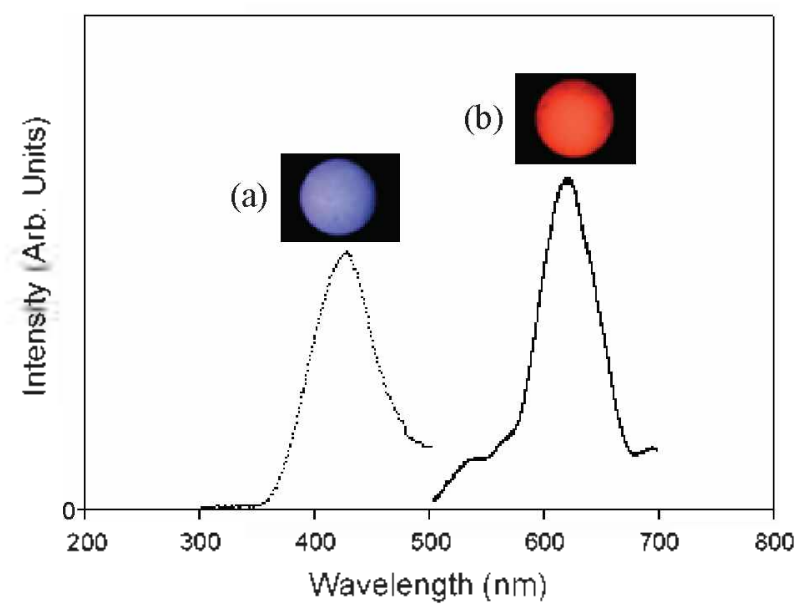

Figure 3. Optical reflection spectra of colloidal crystals and their replicas of titania inverse opal. The peaks and optical images at wavelengths of around (b) $430 \mathrm{~nm}$ and (a) $630 \mathrm{~mm}$ were measured from the colloidal crystals of $260 \mathrm{~mm}$ PS spheres and their titania inverse opals, respectively. The optical microscope inages were viewed around $3 \mathrm{~mm}^{2}$. titania opals with $70 \mathrm{~lm}$ thickness were produced from the colloidal crystal template of $1 \mu \mathrm{m}$ PS spheres after the second deposition. as shown in Figure $2 \mathrm{~d}$.

The optical reflection images and optical reflection spectra of the colloidal crystals and their inverse replicas were also measured, as shown in Figure 3. The inset figures (a and b) show the colors reflected by the PS colloidal cry stals and their titania replicas. Our colloidal cry stals and titania inverse opals exhibit a bright iridescence in reflected light due to the optical diffraction by the crystal multilayer. Because the (111) plane of colloidal crystal is parallel to the substrate, the reflection spectra at normal incidence represents the stop band in the $\Gamma-$ L direction in the Brillouin zone. ${ }^{3 l}$ The peaks appear at around $620 \mathrm{~nm}$ for the colloidal crystals and at $430 \mathrm{~nm}$ for the titania inverse opals. This shift is because the size of air spheres was shrunk relative to the size of the onginal PS spheres dunng heat treatment and the volume fraction of the air cavity was increased in the inverted structure. resulting in lowering the average refractive index. The refractive index of titania can be estimated from Bragg's diffraction law using the reflection peak wavelength $(430 \mathrm{~nm}$ ). the interplanar spacing (175 nm measured from SEM image), and the filling ratio of titania. $8.2 \%$ The filling fraction of titania is around $8 \%$ to $10 \%$ as estima-
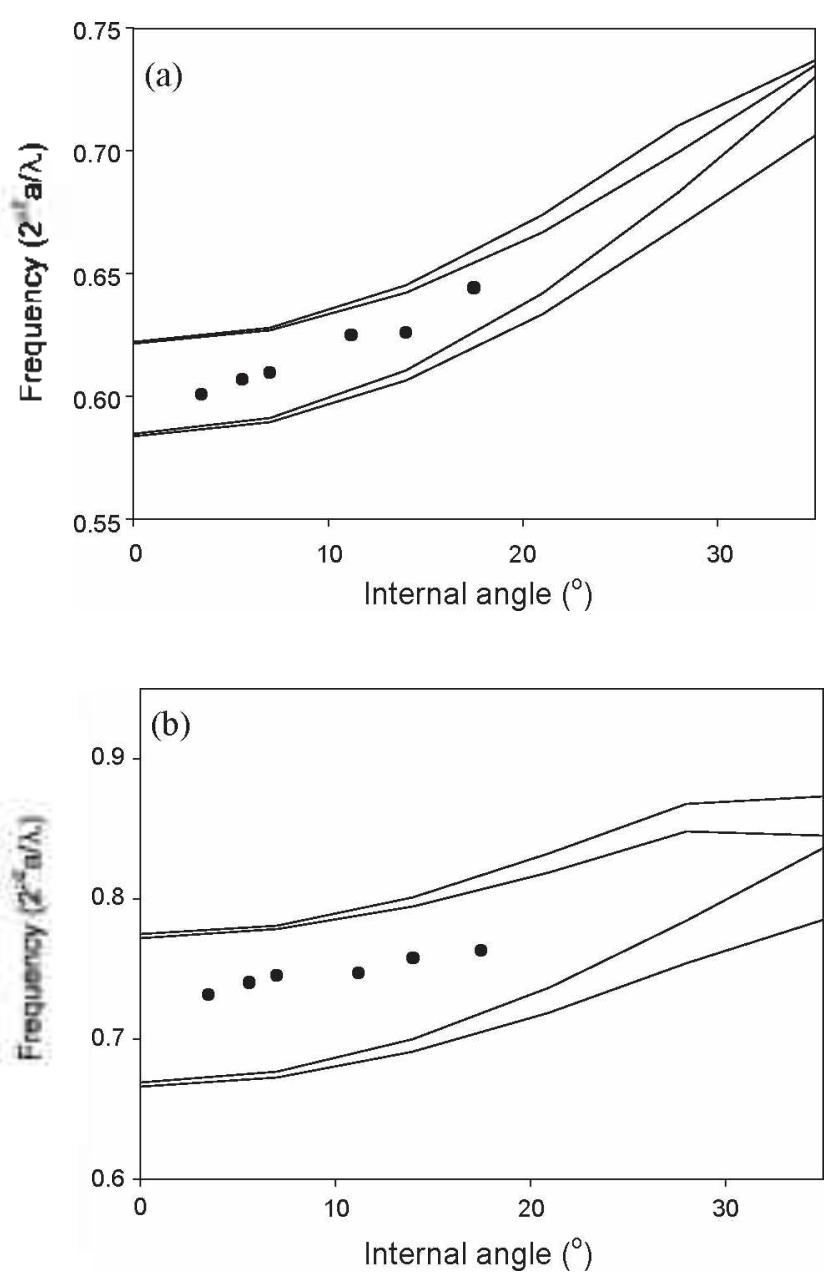

Figure 4 . The reflection peaks measured at different angles correspond to the dots withirn the photonic band diagram. 
ted from the TGA data with densities of $3.84 \mathrm{~g} \mathrm{~cm}^{-3}$ for anatase titania and $1.05 \mathrm{~g} \mathrm{~cm}^{.3}$ for PS spheres. Therefore. by use of Bragg's law the refractive index of titania was estimated to be $2.6 \pm 0.1$, which confirms the anatase phase from XRD measurement. (see Supplementary Information. Figure \$1).

Moreover. the spectra were measured in different directions. When the crystal film was rotated around its vertical axis parallel to the sample growth direction. the diffraction plane moved from the $\mathrm{L}$ point toward the $\mathrm{U}$ point in the Brillouin zone. $^{31}$ We measured the angle-resolved specular measurements with the internal angle of an incident beam varied between of $0^{\circ}$ and $18^{\circ}$. As the incident beam was tilted. the reflection peak position shifted from $630 \mathrm{~nm}$ to $580 \mathrm{~nm}$ for the PS colloidal crystals and from $435 \mathrm{~nm}$ to $417 \mathrm{~nm}$ for the titania inverse opals. In Figure 4, the experimental data are superimposed on the photonic band diagram. We found that the pseudogap toward the $U$ point is wider and more flat in the case of titania inverse opals and the reflection peaks are located within the four lowest-energy band frequencies.

\section{Summary}

We fabricated inverse titania opals via chemical vapor deposition into a polymeric colloidal crystal template. The chemical vapor deposition was achieved at atmospheric pressure and room temperature and produced a uniform and controlled coating of titania over a large area. The thickness of the coated layer increased with the number of titania precursor $\left(\mathrm{TiCl}_{4}\right)$ exposures. Moreover. we found that the moieties remaining after low temperature deposition produce a soft shell on the templates that can reform into a rod-like morphology during heat treatment, due to the surface tension. The titania inverse opals have a filling fraction of $8-10 \%$ and exhibit an anatase phase without the loss of ordered macropore arrangement with a refractive index of $2.6 \pm 0.1$. The angle-resolved reflection exhibits a photonic band stnıcture in different cry stal orientations. Our.

Acknowledgments. JHM acknowledges support by the Sogang University Research Grant of 2008, Korea Research Foundation Grant funded by the Korean Government (MOEH RD) (KRF-2008-313-D00295) and the Manpower Development Program for Energy \& Resources of the Ministry of Knowledge and Economy (2008-E-AP-HM-P-23-0000). The Korea Basic Science Institute is also acknowledged for the measurement of scanning electron microscope.

Supporting Information (XRD pattems of titania inverse opals) are av ailable on request from the correspondence author.

\section{References}

1. Wijnhoven, I.; Vos, W. L. Science 1998, 281. 802

2. Li, X. I.; Qiao, G. I.; Chen, J. R.: Xi, Z. Prog. Chem. 2008, 20. 491.

3. Kim, I. D.; Rothschild, A.; Yang, D. J.; Tuller, H. L. Sensor Actuat A-Phis. 2008, 130,9

4. Lai, Y. K.; Lin, C. I.; Huang. T. Y.; Zhuang, H. F.; Sun, L.; Nguyen, T. Langmitr $2008,24,3867$.

5. Mihi, A.; Calvo, M. E.; Anta, J. A; Miguez, H. J. Phys. Chem. C 2008, 112, 13 .

6. Rodrigues, I; Atienzar, P; Ramiro-Manzano, $\mathrm{F}$; Meseguer, $\mathrm{F}$; Coma, A.: Garcia, H. Photonic. Nanostruct. 2005, 3, 148.

7. Chen, I. I. L.; von Freymann, G.: Kitaev, V:; Ozin, G. A. $d$. Am. Chem. Soc. 2007, 129, 1196.

8. Li, Y. Z.: Kunitake, T.; Fụikawa, S. J. Phys. Chem. B 2006, 110, 13000 .

9. Therriault, D.: Shepherd, R. F; White, S. R.: Lewis, I. A. Adv. Mater: 2005, 17, 395.

10. Qi, M. H.: Lidorikis, E.: Rakich, P. T.; Johnson, S. G.; Toannopoulos, T. D.; Ippen, E. P.: Smith, H. I. Nanure 2004, 429, 538.

11. Camphell, M.; Sharp, D. N.; Harrison, M. T; Denning, R. G.; Turberfield. A. T. Nature 2000, $404,53$.

12. Subramanian, G.; Manoharan, V. N.; Thome, J. D.; Pine, D. T. 4dv : Hater $1999,11,1261$

13. Tiang, P.; Ostojic, G. N.; Narat, R.; Mittleman, D. M.; Colvin, V. L. Adv. Hater: 2001, 13, 389

14. Dong, W. T.; Bongard, H.; Tesche, B.; Marlow; F. Adr Mater: 2002, 14,1457

15. Holland, B. T.: Blantord. C. F.: Do, T.: Stein. A. Chem. Mater. $1999,11,795$.

16. Richel, A.: Tohnson, N. P: McComb, D. W. Appl. Phys Letr. 2000, 76, 1816

17. Wịnhoven, J.: Bechger, L.; Vos, W. L. Chem Aater: 2001, 13, 4486.

18. Gu, Z. Z:; Kubo, S.; Qian, W. P:; Einaga, Y.; Tryk, D. A.; Fuijishima, A.: Sato, O. Langmir 2001, 17,6751

19. Strohm, H.; Lobmant, P. J. Moter Chem. 2004, If, 138.

20. King, .T. S.; Graugnard, E.; Summers, C. J. Adr Moter: 2005, 17 , 1010

21. Gates, B.; Park, S. H.; Xia, Y. N. Adw Mater: 2000, 12, 653.

22. von Frevmann, G.; John, S.; Kitaev, V.; Ozin, G. A. Adv . Haten: 2005, 17,1273

23. Pizem, H.; Sukenik, C. N. Chem. Haten, 2002, 1t, 2476.

24. Havashi, S. Hirai, T. J. Cnst. Growth $1976,36,157$.

25. Yeung, K. S.: Lam, Y. W. Thin Solid Films 1983, 109, 169

26. Bechger. L.: Vos, W. L. Chem. Mater. 2004. 16. 2425

27. Schroden, R. C .: Al-Daous, M.: Blantord, C. F.: Stein, A. Chem. Mater: 2002, 14, 3305 .

28. Dong, W. T.: Bongard, H. T, Marlow; F. Chem Mater 2003, 15, 568 .

29. Manoharan, V. N.; Imhot, A.; Thorne, I. D.: Pine, D. I. Adv. Mater: 2001, 13, 447.

30. Migue, H.; Tetreault, N.; Yang, S. M.; Kitaev, V.; Otin, G. A. Adv Waten $2003,15,597$.

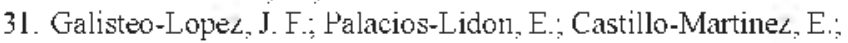
Lopez, C. Phys. Rev. B 2003, 68, 115109. 